Please do not remove this page

RMIT

UNIVERSITY

\title{
Long-range dipolar order and dispersion forces in polar liquids
}

Besford, Quinn; Christofferson, Andrew; Liu, Maoyuan; Yarovsky, Irene

https://researchrepository.rmit.edu.au/esploro/outputs/9921861729501341/filesAndLinks?institution=61RMIT_INST\&index=null

Besford, Q., Christofferson, A., Liu, M., \& Yarovsky, I. (2017). Long-range dipolar order and dispersion forces in polar liquids. Journal of Chemical Physics, 147(19), 1-8. https://doi.org/10.1063/1.5005581 Document Version: Published Version

Published Version: https://doi.org/10.1063/1.5005581

Repository homepage: https://researchrepository.rmit.edu.au (C) 2017 Author(s)

Downloaded On 2023/04/26 10:00:51 +1000

Please do not remove this page 
Thank you for downloading this document from the RMIT Research Repository.

The RMIT Research Repository is an open access database showcasing the research outputs of RMIT University researchers.

RMIT Research Repository: http://researchbank.rmit.edu.aul

\section{Citation:}

Besford, Q, Christofferson, A, Liu, M and Yarovsky, I 2017, 'Long-range dipolar order and dispersion forces in polar liquids', Journal of Chemical Physics, vol. 147, no. 19, pp. 1-8.

See this record in the RMIT Research Repository at:

https://researchbank.rmit.edu.au/view/rmit:47234

Version: Published Version

Copyright Statement:

(C) 2017 Author(s)

\section{Link to Published Version:}

https://dx.doi.org/10.1063/1.5005581 


\section{Long-range dipolar order and dispersion forces in polar liquids}

Cite as: J. Chem. Phys. 147, 194503 (2017); https://doi.org/10.1063/1.5005581

Submitted: 19 September 2017 . Accepted: 26 October 2017 . Published Online: 16 November 2017

Quinn Alexander Besford (D), Andrew Joseph Christofferson (D, Maoyuan Liu, and Irene Yarovsky (D)

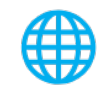

View Online

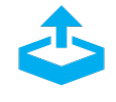

Export Citation

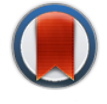

\section{ARTICLES YOU MAY BE INTERESTED IN}

Communication: Mean-field theory of water-water correlations in electrolyte solutions The Journal of Chemical Physics 146, 181103 (2017); https://doi.org/10.1063/1.4983221

Building better water models using the shape of the charge distribution of a water molecule

The Journal of Chemical Physics 147, 194103 (2017); https://doi.org/10.1063/1.4986070

Perspective: Dissipative particle dynamics

The Journal of Chemical Physics 146, 150901 (2017); https://doi.org/10.1063/1.4979514

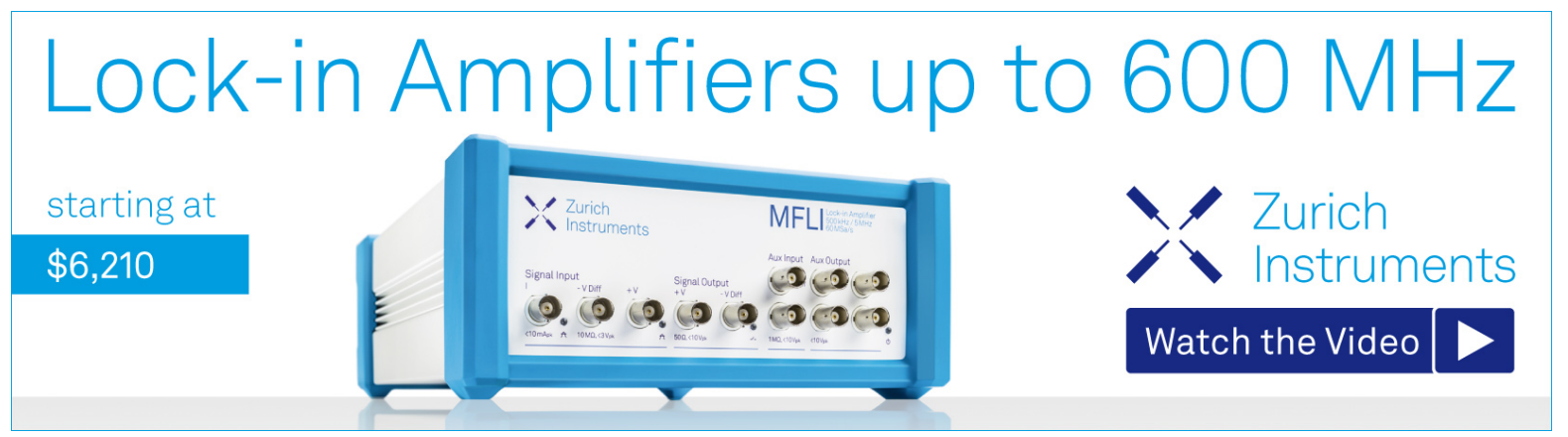

J. Chem. Phys. 147, 194503 (2017); https://doi.org/10.1063/1.5005581 


\title{
Long-range dipolar order and dispersion forces in polar liquids
}

\author{
Quinn Alexander Besford, ${ }^{1, a)}$ Andrew Joseph Christofferson, ${ }^{2}$ Maoyuan Liu, ${ }^{3}$ \\ and Irene Yarovsky² \\ ${ }^{1}$ Department of Chemical Engineering, The University of Melbourne, Victoria 3010, Australia \\ ${ }^{2}$ School of Engineering, RMIT University, Victoria 3001, Australia \\ ${ }^{3}$ School of Chemistry, The University of Melbourne, Victoria 3010, Australia
}

(Received 19 September 2017; accepted 26 October 2017; published online 16 November 2017)

\begin{abstract}
Complex solvation phenomena, such as specific ion effects, occur in polar liquids. Interpretation of these effects in terms of structure and dispersion forces will lead to a greater understanding of solvation. Herein, using molecular dynamics, we probe the structure of polar liquids through specific dipolar pair correlation functions that contribute to the potential of mean force that is "felt" between thermally rotating dipole moments. It is shown that unique dipolar order exists at separations at least up to $20 \AA$ for all liquids studied. When the structural order is compared with a dipolar dispersion force that arises from local co-operative enhancement of dipole moments, a strong agreement is found. Lifshitz theory of dispersion forces was compared with the structural order, where the theory is validated for all liquids that do not have significant local dipole correlations. For liquids that do have significant local dipole correlations, specifically liquid water, Lifshitz theory underestimates the dispersion force by a factor of 5-10, demonstrating that the force that leads to the increased structure in liquid water is missed by Lifshitz theory of van der Waals forces. We apply similar correlation functions to an ionic aqueous system, where long-range order between water's dipole moment and a single chloride ion is found to exist at $20 \AA$ of separation, revealing a long-range perturbation of water's structure by an ion. Furthermore, we found that waters within the 1st, 2nd, and 3rd solvation shells of a chloride ion exhibit significantly enhanced dipolar interactions, particularly with waters at larger distances of separation. Our results provide a link between structures, dispersion forces, and specific ion effects, which may lead to a more robust understanding of solvation. Published by AIP Publishing. https://doi.org/10.1063/1.5005581
\end{abstract}

\section{INTRODUCTION}

Complex solvation phenomena occur in polar liquids, which include specific ion effects in aqueous ${ }^{1,2}$ and nonaqueous systems, ${ }^{3}$ solvophobic effects that reduce the solubility and drive the coalescence of apolar species immersed in polar liquids ${ }^{4,5}$ as well as the high surface energies of polar liquids. ${ }^{6}$ The mechanistic driving force behind these phenomena is not well understood but is likely related to the perturbation of the liquid's structure by solutes and interfaces ${ }^{7}$ and how that enhances or diminishes intermolecular correlations. The complexity of liquid water, as one example, is thought to be due to a combination of small size and a distinct polar charge distribution within the water molecule. ${ }^{8}$ For polar liquids, and water in particular, there exists strong orientation-dependent attractive interactions which can have a substantial structural influence. ${ }^{9,10}$ Given the importance of such systems to industry and biology, there is a great interest in the elucidation of the intermolecular structure of polar liquids.

Polar liquids exhibit more structural order than nonpolar liquids, resulting from the electric fields around each molecule's dipole moment, which causes a hindering of the

a)Electronic mail: quinn.besford@gmail.com rotation of neighbouring dipole moments, relative to itself. ${ }^{11,12}$ Oster and Kirkwood ${ }^{13}$ suggested that hindered molecular rotation due to local correlations in the orientations of neighbouring molecules might augment or diminish the contributions of individual molecular dipoles to the macroscopic dielectric constant of the liquid. Kirkwood ${ }^{14}$ showed that this contribution is as significant as the molecular dipole moment alone. Other macroscopic properties, such as the long-range forces that are "felt" between surfaces immersed in polar liquids, ${ }^{15}$ are also likely a result of local interactions that have long-range influence. These latter phenomena are generally attributed to dispersion forces. ${ }^{16,17}$

Spectroscopic and neutron scattering methods have successfully been used to analyze local liquid structure, such as spatial density functions, ${ }^{18}$ hydrogen bonding dynamics, ${ }^{19}$ and radial distribution functions $[\mathrm{g}(\mathrm{r})] .{ }^{20}$ In particular, second harmonic scattering methods have revealed information on the structure at interfaces and around solutes in great detail, where the method can measure coherent and incoherent scattered light, which contains contributions from cooperative scattering of neighbouring molecules whose positions and orientations are correlated, and those from different regions of the liquid, respectively. ${ }^{21-23}$ However, molecular dynamics (MD) simulations allow for a greater probe of long-range structure from knowledge of the exact locations and orientations of each molecule. ${ }^{24,25}$ MD methods, often in combination 
with experiment, have provided valuable information on local structuring in liquids, particularly around solutes and interfaces. ${ }^{26-28}$ However, the analysis of long-range structure from both MD simulations and experimental methods crucially depends on the type of order parameter that is used to analyze the structure [e.g., $g(r)$, tetrahedral order, hydrogen bonding]. Consequently, such methods can only provide information on long-range order in liquids if the functional form of the correlations that lead to the order is known. For example, pairdistribution functions obtained from MD, theoretical predictions, or scattering experiments only provide a partial description of the higher dimensional intermolecular correlation function that depends on both the positions and orientations of the molecules. $^{29}$

Analysis of dipolar correlation functions have revealed long-range order in liquid water, ${ }^{30}$ and the perturbation of this order by simple and ionic solutes ${ }^{31,32}$ and interfaces. ${ }^{33}$ The dominating long-range order in liquid water has generally been found in the longitudinal part of the dipole-dipole orientational correlations, which has been suggested to be due to an underlying network of fluctuating hydrogen bonds. ${ }^{34}$ Using density functional theory, Liu and $\mathrm{Wu}^{29}$ have shown that liquid water has orientational correlations at separations of about $40 \AA$, and the angular correlation function attenuates in an oscillatory manner. Given that water can perturb the dynamics of biomolecular interactions directly in the first solvation shell and indirectly at several solvation shells of separation, ${ }^{35}$ an understanding of the long-range orientational correlations in water is necessary for understanding solvation in biology. Specifically, linking this structural order to the intermolecular forces within water and its perturbation by solutes.

Liquid water is the most studied liquid due to its importance in biology, but it is not immediately clear to what extent other polar liquids exhibit long-range order and how similar or different it is from liquid water. In order to gain a better understanding of the molecular role of the orientational correlations in solvation, the long-range structure of polar liquids needs to be directly quantified and further analysed with respect to intermolecular forces in order to elucidate how it relates to the dispersion forces. Only by relating liquid structure to dispersion forces within the liquid can the role of solvation in macroscopic phenomena within polar liquids be better interpreted.

Herein, we quantify dipolar order in various polar liquids through a specific set of pair correlation functions that describe correlations in orientations between thermally rotating permanent dipole moments. These dipole correlations have recently been shown to capture the hydrophobic effect, i.e., the signature, temperature dependent, solvation entropy of nonpolar molecules in water. ${ }^{36}$ The orientational order of dipoles manifests as a local co-operative enhancement of dipole moments and is associated with a potential of mean force (PMF). This orientational order can also be described macroscopically as a dispersion force between dipoles via a mean field treatment. We directly compare the simulated orientational PMF with a macroscopic dipolar dispersion force for a range of polar liquids, where a strong agreement is found. Our results point toward a direct relationship between orientational dipolar order in polar liquids and dipolar dispersion forces.

\section{THEORY}

The angular-dependent radial distribution function (RDF), $g^{i j}$, between two dipole moments of a liquid, $\mu_{1}$ of molecular species $i$ and $\boldsymbol{\mu}_{2}$ of molecular species $j$ separated by $\boldsymbol{r}$, may be given in terms of the angular dependent PMF $\mathrm{as}^{36}$

$$
g^{i j}\left(\boldsymbol{r}, \boldsymbol{\mu}_{1}, \boldsymbol{\mu}_{2}\right)=\exp \left(-\beta \mathcal{W}^{i j}\left(\boldsymbol{r}, \boldsymbol{\mu}_{1}, \boldsymbol{\mu}_{2}\right)\right),
$$

where $\beta=\left(k_{B} T\right)^{-1}, T$ is the absolute temperature, $k_{B}$ Boltzmann's constant, and $\mathcal{W}^{i j}$ is the PMF. The expansion of the angular-dependent functions in Eq. (1) in the basis of rotational invariants, ${ }^{37} \Phi_{l_{1} l_{2} l}\left(\hat{\boldsymbol{r}}, \hat{\boldsymbol{\mu}}_{1}, \hat{\boldsymbol{\mu}}_{2}\right)$, gives

$$
\begin{aligned}
& -\beta \mathcal{W}_{S}^{i j}(r)-\sum_{l_{1}} \sum_{l_{2}} \sum_{l \geq\left|l_{1}-l_{2}\right|}^{l_{1}+l_{2}} \beta \mathcal{W}_{l_{1} l_{2} l}^{i j}(r) \Phi_{1_{1} l_{2} l} \\
& =\log \left(g_{S}^{i j}(r)+\sum_{l_{1}} \sum_{l_{2}} \sum_{l \geq\left|l_{1}-l_{2}\right|}^{l_{1}+l_{2}} h_{l_{1} l_{2} l}^{i j}(r) \Phi_{1_{1} l_{2} l}\right),
\end{aligned}
$$

where $h_{l_{1} l_{2} l}^{i j}$ is an expansion coefficient describing preferential modes of dipole orientations selected by $\Phi_{l_{1} l_{2} l}$ and the subscript " $S$ " denotes the term independent of dipole orientation. Gray and Gubbins ${ }^{38}$ describe the allowed values of the indices in the $h_{l_{1} l_{2} l}$ dipolar pair correlation functions. The contribution of dipole correlations $h_{l_{1} l_{2} l}^{i j}$ to the angular averaged PMF, $\omega^{i j}(r)$ $=-k_{B} T \log \left(g_{S}^{i j}(r)\right)$, is expressed as

$$
\begin{aligned}
\omega^{i j}(r)= & \mathcal{W}_{S}^{i j}(r) \\
& -k_{B} T\left|\log \left(1+\sum_{l_{1}} \sum_{l_{2}} \sum_{l \geq\left|l_{1}-l_{2}\right|}^{l_{1}+l_{2}} \frac{h_{l_{1} l_{2} l}^{i j}(r)}{g_{S}^{i j}(r)} \Phi_{1_{1} l_{2} l}\right)\right|_{\boldsymbol{\mu}_{1}, \boldsymbol{\mu}_{2}} .
\end{aligned}
$$

The expansion of the logarithm in Eq. (3) to the second order in rotational invariants for correlations between pairs of solvent molecules, i.e., $i=j=s$, yields

$$
\begin{aligned}
\omega^{s s}(r) & =\mathcal{W}_{S}^{s s}(r)+\phi(r)+O\left(\left(h_{l_{1} l_{2} l}^{s s}(r)\right)^{3} /\left(g_{S}^{s s}(r)\right)^{3}\right), \\
\phi(r) & =-k_{B} T \frac{1}{2\left(g_{S}^{s s}(r)\right)^{2}} \sum_{l_{1}} \sum_{l_{2}} \sum_{l \geq\left|l_{1}-l_{2}\right|}^{l_{1}+l_{2}}\left(h_{l_{1} l_{2} l}^{s s}(r)\right)^{2},
\end{aligned}
$$

where $\phi(r)$ is an orientation-independent contribution to the PMF arising from orientational correlation between dipoles. Note that $h_{l_{1} l_{2} l}(r)$ can be calculated from MD simulations via $h_{l_{1} l_{2} l}(r)=\langle\Phi\rangle_{\mu 1, \mu 2,|\mathbf{r}|=r}$ and that the $\phi(r)$ computed from the simulated $h_{l_{1} l_{2} l}(r)$ is denoted $\phi_{M D}(r)$ for clarity. The first few real-valued rotational invariants are

$$
\begin{gathered}
\Phi_{1,1,0}\left(\hat{\boldsymbol{r}}, \hat{\boldsymbol{\mu}}_{1}, \hat{\boldsymbol{\mu}}_{2}\right)=-\sqrt{3} \hat{\boldsymbol{\mu}}_{1} \cdot \hat{\boldsymbol{\mu}}_{2}, \uparrow \uparrow \text { or } \rightarrow \rightarrow, \\
\Phi_{1,1,2}\left(\hat{\boldsymbol{r}}, \hat{\boldsymbol{\mu}}_{1}, \hat{\boldsymbol{\mu}}_{2}\right) \\
=-\sqrt{\frac{3}{2}}\left(3\left(\hat{\boldsymbol{\mu}}_{1} \cdot \hat{\boldsymbol{r}}\right)\left(\hat{\boldsymbol{\mu}}_{2} \cdot \hat{\boldsymbol{r}}\right)-\left(\hat{\boldsymbol{\mu}}_{1} \cdot \hat{\boldsymbol{\mu}}_{2}\right)\right), \uparrow \downarrow \text { or } \rightarrow \rightarrow,
\end{gathered}
$$




$$
\Phi_{1,0,1}\left(\hat{\boldsymbol{r}}, \hat{\boldsymbol{\mu}}_{1}\right)=\sqrt{3} \hat{\boldsymbol{\mu}}_{1} \cdot \hat{\boldsymbol{r}}, \rightarrow
$$

where $\left(l_{1} l_{2} l\right)=(110)$ gives the tendency for dipoles to align parallel or head-to-tail, $\left(l_{1} l_{2} l\right)=(112)$ gives the tendency for dipoles to adopt low energy configurations, and $\left(l_{1} l_{2} l\right)$ $=(101)$ gives the tendency for a dipole to point toward another molecule. Since $\phi(r)$ is always negative, it produces an attractive force between pairs of polar molecules. The slowest decaying correlation function in Eq. (5) is the $h_{112}$ function, ${ }^{39}$ which has a limiting form of

$$
\lim _{r \rightarrow \infty} h_{112}(r)=\sqrt{\frac{2}{3}} \frac{(\epsilon-1)^{2}}{4 \pi y \rho \epsilon} \frac{1}{r^{3}},
$$

where $y=4 \pi \rho \mu^{2} / 9 k_{B} T, \rho$ is the number density, and $\epsilon$ is the static permittivity. It is this function that dominates $\phi(r)$ at large distances. Following the analysis of Ref. 36, the asymptotic form of $\phi(r)$ may be expressed in terms of the macroscopic dielectric properties of a liquid as

$$
\begin{aligned}
\phi_{\infty}(r) & =\lim _{r \rightarrow \infty} \phi(r)=-\frac{k_{B} T}{2}\left(\lim _{r \rightarrow \infty} h_{112}(r)\right)^{2} \\
& =-k_{B} T \frac{27 g_{K}^{2}(\epsilon-1)^{2}}{16 \pi^{2}(1+2 \epsilon)^{2} \rho^{2}} \frac{1}{r^{6}} \\
& =-k_{B} T \frac{27 g_{K}^{2}}{64 \pi^{2}}\left[1+\mathcal{O}\left(\frac{1}{\epsilon}\right)\right] \frac{1}{\rho^{2} r^{6}},
\end{aligned}
$$

where $g_{K}$ is the Kirkwood $g$-factor, which describes the average alignment of dipoles, given as

$$
g_{K}=\frac{\left\langle|\boldsymbol{M}|^{2}\right\rangle}{N \mu^{2}},
$$

where $\left\langle|\boldsymbol{M}|^{2}\right\rangle$ is the mean-squared total dipole moment of $N$ molecules. Equation (11) is an exact result for a fluid of rigid dipoles, the strength of which is proportional to $g_{K}^{2}$. Recently, Liu et al. ${ }^{36}$ reported that the hydrophobic effect, i.e., the signature, temperature dependent, solvation entropy of nonpolar molecules in water, is largely due to the interactions between dipole moments that are found in Eqs. (5) and (11). Herein, we provide direct comparisons between liquid structure, as found in $\phi_{M D}(\mathrm{r})$, and intermolecular forces, as found in $\phi_{\infty}(\mathrm{r})$, for various polar liquids and an ionic aqueous system.

\section{MOLECULAR DYNAMICS}

\section{A. Model construction}

The polar liquids studied in this work were water, methanol, 50\% water 50\% methanol, ethanol, 1-propanol, and 1-butanol. The polar aprotic liquids studied in this work were acetone, acetonitrile, and $N, N$-dimethylformamide. Cubic simulation boxes comprising 2112 molecules of water and 960 solvent molecules for all other systems were constructed with initial box dimensions based on the experimental density of the solvent, approximately $40 \times 40 \times 40 \AA^{3}$. For the simulation of a chloride ion in water, a single chloride ion was placed in the centre of a box of 2112 water molecules. An additional simulation of a single sodium and chloride ion in water (2112 molecules) was also performed to check the effect of system charge neutrality. For this simulation, the sodium and chloride ions were started at the furthermost separations. Larger boxes of approximately $100 \times 100 \times 100 \AA^{3}$ comprising 33000 water molecules and 7680 1-butanol molecules, respectively, were also constructed by the same method.

\section{B. Simulation}

For all fixed-charge force field MD simulations, parameters from the Condensed-phase Optimized Molecular Potentials for Atomistic Simulation Studies (COMPASS) force field ${ }^{40}$ were applied. We have previously used this force field to study systems with water at hydrophobic ${ }^{41}$ and hydrophilic ${ }^{42}$ interfaces. For the Lennard-Jones parameters of the chloride ion in the 9-6 functional form, ${ }^{40}$ we applied a sigma value of $5.3 \AA$ and epsilon of $0.09 \mathrm{kcal} / \mathrm{mol}$, based on a comparison of radial distribution functions (RDFs) and self-diffusion constants from MD simulations with experimental values. ${ }^{43,44} \mathrm{We}$ chose the COMPASS force field for this work because all of the solvents studied (water, alcohols up to propanol, and all three of the polar aprotic solvents) were part of the original force field validation set and show a good agreement between experimental and simulated densities and heats of vaporization. ${ }^{40}$ For simulations of water and alcohol, we applied the published parameters of COMPASS in the open-source MD code Large-Scale Atomic/Molecular Massive Parallel Simulator (LAMMPS). ${ }^{45}$ For the simulations of aprotic solvents, we applied the COMPASS force field implemented in the Forcite MD code. ${ }^{46}$ Electrostatic interactions beyond a 15.5 $\AA$ cutoff were evaluated with the particle-particle-particlemesh (PPPM) summation method with an accuracy of $10^{-5}$ $\mathrm{kcal} / \mathrm{mol}$, and van der Waals interactions were assessed with an atom-based summation using a $15.5 \AA$ cutoff and tail correction. MD simulations were run in the isothermal-isobaric $(N p T)$ ensemble, using the Nosé-Hoover thermostat and barostat to maintain a temperature of $298 \mathrm{~K}$ and a pressure of $1 \mathrm{~atm}$, with a time step of $1 \mathrm{fs}$, and an output frequency of 20 ps.

All simulations were run for $25 \mathrm{~ns}$, with equilibration for the first $5 \mathrm{~ns}$, and analysis performed on the final $20 \mathrm{~ns}$. The simulation of the chloride ion in water was run for a total of $105 \mathrm{~ns}$, with analysis performed on the final $100 \mathrm{~ns}$. For the polarizable solvents, we used the polarizer tool in LAMMPS ${ }^{47}$ to apply the SWM4-NDP ${ }^{48}$ and mod-CHARMM/PARAM$22^{49}$ force field parameters to the existing water and ethanol boxes, respectively. The simulations were run in LAMMPS following the procedure outlined by Dequidt et al.,${ }^{47}$ with all other settings identical to the fixed-charge force field simulations.

\section{RESULTS}

The interaction of two thermally rotating dipole moments, as quantified by the pair correlations in $\phi(r)$, may be calculated from simulated trajectories of polar molecules. We performed MD simulations of various polar liquids, including water, with the COMPASS $^{40}$ force field and the $(N p T)$ ensemble, with initial cubic box dimensions in excess of $40 \AA$ in each cardinal direction, containing 960 molecules for non-water systems and 2112 molecules for water systems. 
The pair correlations that are quantified by MD trajectories we label as $\phi_{M D}(\mathrm{r})$, to distinguish them from the asymptotic form, which are shown for all liquids in Fig. 1 (dark blue dots), along with the respective RDFs (red dashed lines). For all liquids simulated, long-range correlations between permanent dipole moments exist at molecular separations at least up to $20 \AA$. Zhang and Galli ${ }^{32}$ have shown that dipolar order, as observed through a radial dipole-dipole spatial correlation function, varies considerably depending on the size of the simulated system. We validated that the $\phi_{M D}(\mathrm{r})$ function is consistent regardless of system size for pure water and pure 1-butanol correlations by comparing a system of 2112 molecules to one of 33000 molecules and of 960 molecules7680 molecules, respectively, where no significant difference was observed between the systems (see supplementary material).

The short-range oscillations in $\phi_{M D}(\mathrm{r})$ appear to qualitatively match the position of the oscillations in the $g(\mathrm{r})$, though the $\phi_{M D}(\mathrm{r})$ correlations clearly exist at greater length scales than the $g(\mathrm{r})$. Dipolar orientational correlations have previously been observed at the $\mathrm{nm}$ scale by MD simulations and density functional theory (DFT) calculations for liquid water. ${ }^{50}$ Mathias and Tavan ${ }^{30}$ have used unique pair correlation functions to show that TIP3P liquid water ${ }^{51}$ exhibits solvation shell-like structures up to distances of $12.5 \AA$, in agreement with Fig. 1(a). However, we show not only that dipolar order exists over large distances but also how the preferential orientations of dipole moments found in the $h_{l_{1} l_{2} l}^{s s}$ correlation functions contribute to $\phi_{M D}(\mathrm{r})$ and ultimately to the free energy of the fluid. ${ }^{36}$
Comparing the different liquids, noticeable differences in the oscillations in the correlations exist, highlighting differences in dipolar order for each of the liquids that are likely related to molecular size and shape. This is consistent with Oster and Kirkwood's work, ${ }^{13}$ which showed that the nature of orientational correlations between thermally rotating dipole moments is not determined only by the molecular dipoles alone but also by the location of the permanent dipole moment within the polar molecule. In particular, $\phi_{M D}(\mathrm{r})$ for water is very well converged at distances approaching $20 \AA$, whereas those for DMF and acetonitrile still exhibit clear oscillations at these distances. For 1-butanol, 1-propanol, and methanol, the functions are not as well converged in the same simulation time, which may be indicative of less structuring in these liquids.

The asymptotic result for the same dipolar correlations, $\phi_{\infty}(\mathrm{r})$, as given by Eq. (11), is overlaid on top of the other functions in Fig. 1 (solid red lines). Comparing the initial spike and the long-range tail in $\phi_{M D}(\mathrm{r})$ to the asymptotic $\phi_{\infty}(\mathrm{r})$, it is seen that the asymptotic form matches the decay in $\phi_{M D}(\mathrm{r})$ reasonably well. The greatest agreement is found for water and acetone, whereas the poorest agreement is found for acetonitrile. This latter result may stem from another force that influences the macroscopic dielectrics; this may include those stemming from dipole-quadrupole and quadrupole-quadrupole interactions. However, for all other liquids studied, a reasonable agreement is found, and furthermore the comparisons highlight that the $\phi_{M D}(\mathrm{r})$ interaction decays as $1 / \mathrm{r}^{6}$, as does any other van der Waals force. This shows that the $\phi(r)$ interaction is a dipolar dispersion force. It is important to note that there
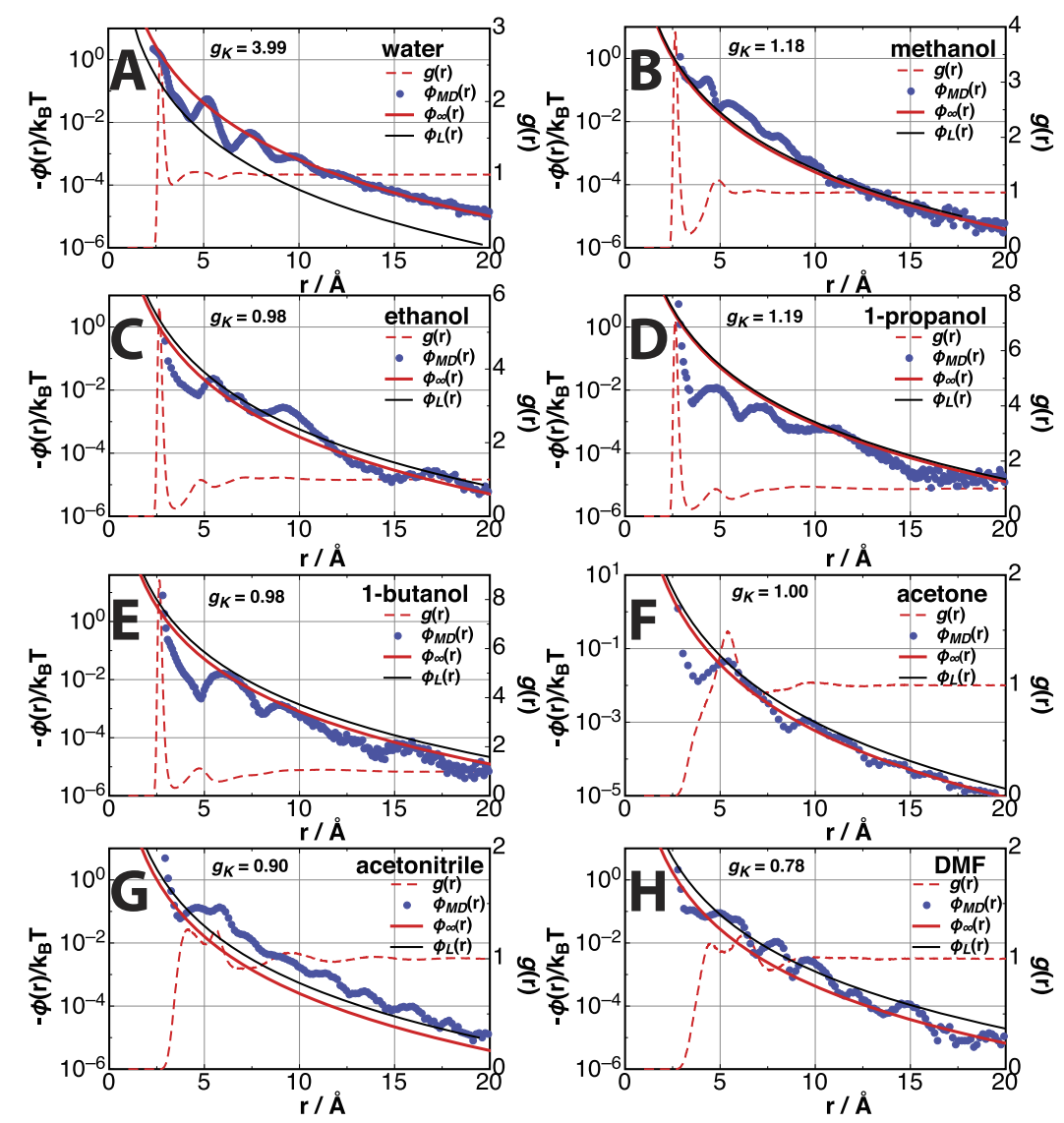

FIG. 1. Full form $\phi_{M D}(\mathrm{r})$ functions calculated from MD trajectories generated with the COMPASS force field ${ }^{40}$ for water (a), methanol (b), ethanol (c), 1-propanol (d), 1-butanol (e), acetone (f), acetonitrile (g), and $N, N$ dimethylformamide (DMF) (h) along with the asymptotic functions, $\phi_{\infty}(\mathrm{r})$, the Lifshitz theory prediction, $\phi_{L}(\mathrm{r})$, the radial distribution functions, $g(\mathrm{r})$, and the Kirkwood $g$-factor, $g_{K}$. Note that all calculations are performed on the simulated liquid dielectric properties. 
are no free parameters for these calculations of dipolar order. The $\phi_{M D}(\mathrm{r})$ results are based only on simulated MD trajectories, and the $\phi_{\infty}(\mathrm{r})$ results are based only on the simulated macroscopic dielectric properties for the same simulated systems. This key result suggests that the $\phi_{\infty}(\mathrm{r})$ dipolar dispersion interaction is the driving force for the long-range structural features of the polar liquids in Fig. 1.

Lifshitz theory ${ }^{52,53}$ can be used to estimate dispersion forces from knowledge of the bulk dielectric spectra of the materials from which the interacting bodies are made. ${ }^{54}$ The theory has successfully been used to estimate the properties of non-polar systems $;{ }^{55}$ however, the theory often fails for liquid water. $^{6}$ We look to compare the structure found in $\phi_{M D}(\mathrm{r})$, and the dispersion force from $\phi_{\infty}(r)$, with Lifshitz theory estimates of van der Waals forces between molecules in the polar liquids. It is crucial to note that $\phi_{M D}(\mathrm{r})$ and $\phi_{\infty}(\mathrm{r})$ are in no way dependent on Lifshitz theory.

Following the discussion by Israelachvili6 (see Chap. 13), the approximate non-retarded Hamaker constant for two identical phases interacting across a vacuum may be given as

$$
A \approx \frac{3}{4} k_{B} T\left(\frac{\epsilon-1}{\epsilon+1}\right)^{2}+\frac{3 h v_{e}}{16 \sqrt{2}} \frac{\left(n^{2}-1\right)^{2}}{\left(n^{2}+1\right)^{3 / 2}},
$$

where $h$ is Planck's constant, $v_{e}$ is the main electronic absorption frequency in the $\mathrm{UV}$, and $n$ is refractive index of the medium in the visible region. The first term in Eq. (14) corresponds to the zero-frequency energy of the van der Waals interaction, which includes the Keesom and Debye dipolar contributions. The second term gives the dispersion energy that includes London energy contributions. Here, we only consider the effective dispersion force that arises from orientational order between permanent dipole moments. We make no attempt to compare against electronic dispersion and London dispersion forces; therefore, only the first term in Eq. (14) is needed to calculate the dipolar dispersion van der Waals interaction (i.e., the zero-frequency dielectric contribution).

The van der Waals interaction between two molecules in a liquid may be expressed as

$$
\phi_{v d w}=-\frac{C}{r^{6}}
$$

where

$$
C=\frac{A}{\pi^{2} \rho^{2}} .
$$

Substituting the first term of Eq. (14) into Eq. (16), the approximate Lifshitz expression for the zero-frequency nonretarded Lifshitz interaction between two permanent dipole moments, from Eq. (15), may be written as

$$
\phi_{L}(r) \approx-k_{B} T \frac{3}{4} \frac{(\epsilon-1)^{2}}{\pi^{2} \rho^{2}(\epsilon+1)^{2}} \frac{1}{r^{6}}
$$

It should be noted that Eq. (17) is an approximate expression and is non-vanishing at short or long length-scales, where it is expected short-range interactions will disappear at separations approaching molecular dimensions and at large length-scales where retardation effects become apparent. We only provide Lifshitz theory here for comparative purposes to the structure found in $\phi_{M D}(\mathrm{r})$ and to the decay of $\phi_{\infty}(\mathrm{r})$. The differences between Eqs. (11) and (17) should be noted; primarily, the lack of $g_{K}$ in the Lifshitz expression. It is this local correlation of dipole moments that is missed by Lifshitz theory due to the assumption of a structureless dielectric continuum.

We compare Lifshitz theory for dipolar dispersion forces, $\phi_{L}$, at short-length scales to $\phi_{M D}(\mathrm{r})$ and $\phi_{\infty}(\mathrm{r})$ in Fig. 1 (red solid lines). Lifshitz theory matches the $\phi_{M D}(\mathrm{r})$ correlations for most liquids, except for liquid water, where it is about a factor of 10 too weak. Out of all the liquids in Fig. 1, liquid water is the only liquid with a significant $g_{K}$. Therefore, the reason for the difference in liquid water is likely the result of the neglect of local dipole correlations in Lifshitz theory. It is therefore this local dipolar interaction that leads to an enhanced $1 / r^{6}$ attraction in liquid water and that is correctly handled through $\phi_{\infty}(\mathrm{r})$. It is likely that this neglect is also the source of the theory's failure for other water systems, such as for the "hydrophobic interaction" between non-polar surfaces immersed in water, ${ }^{56-58}$ where Lifshitz theory also underestimates the force by about an order of magnitude. For other liquids, the $\phi_{L}(\mathrm{r})$ interaction matches the $\phi_{M D}(\mathrm{r})$ structure reasonably well, where it is marginally greater than the $\phi_{\infty}(\mathrm{r})$ interaction, which may indicate where Lifshitz theory incorporates an additional dipolar interaction than those that are found in both $\phi_{M D}(\mathrm{r})$ and $\phi_{\infty}(\mathrm{r})$. However, the comparisons between $\phi_{M D}(\mathrm{r})$ and $\phi_{L}(\mathrm{r})$ validate Lifshitz theory for polar liquids that have $g_{K} \approx 1$. It must be noted that before the $\phi_{\infty}(\mathrm{r})$ interaction can be applied to macroscopic systems, the modulation of the interaction by ions ${ }^{4}$ must first be fully characterized and understood.

The effects of having explicit terms for molecular polarization in the force field on the $\phi(r)$ interactions was investigated by simulating liquid water and ethanol with the SWM4$\mathrm{NDP}^{48}$ and mod-CHARMM/PARAM- $22^{49}$ polarizable force fields, respectively. The $\phi_{M D}(\mathrm{r})$ interaction for the polarizable water model (Fig. 2) agrees well with the COMPASS force field [Fig. 1(a)] and also the SPC/E and TIP4P water models. ${ }^{36}$ Furthermore, asymptotic $\phi_{\infty}(\mathrm{r})$ also match. For the polarizable ethanol, we find that the dipole correlations are enhanced in comparison with the COMPASS force field [Fig. 1(c)], which is a consequence of the polarizable ethanol having $g_{K} \approx 3$, in better agreement with experiment ${ }^{59}\left(g_{K}=2.9\right)$, and compared to COMPASS ethanol $\left(g_{K} \approx 1\right)$. Furthermore, Lifshitz theory underestimates the magnitude of the dipolar dispersion force for both liquids, consistent with the

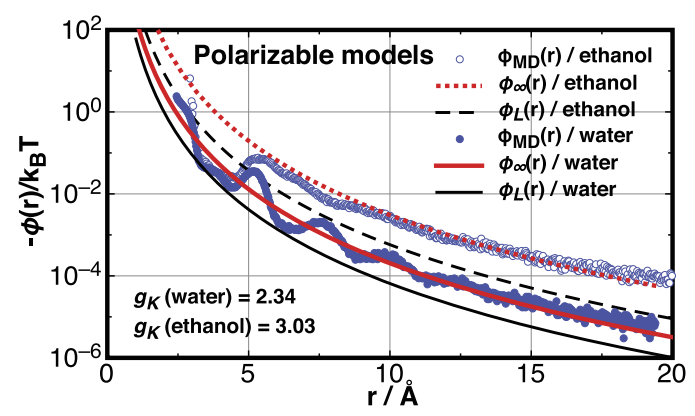

FIG. 2. A comparison of the full dipolar correlations $\phi_{M D}(\mathrm{r})$ for polarizable models of both water (SWM4-NDP ${ }^{48}$ ) and of ethanol (modCHARMM/PARAM-22 ${ }^{49}$ ) along with their asymptotic functions $\phi_{\infty}(\mathrm{r})$, and the Lifshitz theory prediction $\phi_{L}(\mathrm{r})$. 
theory's neglect of $g_{K}$. Asymptotic $\phi_{\infty}(\mathrm{r})$ captures both liquids reasonably well, albeit over estimating the ethanol interaction marginally.

The systems studied thus far have revealed unique structural features for homogenous liquids and the relative strength of dipolar dispersion forces within them. Analysis of how these features change when liquids are mixed may offer further insight into the nature of dipolar correlations in solvation and miscibility. Mixtures of water and methanol are particularly interesting from a structural viewpoint as both liquids are highly associating but exhibit different patterns of hydrogen bonding, ${ }^{60}$ and when the two liquids are mixed, the entropy of the system increases far less than expected for an ideal solution of randomly mixed molecules. ${ }^{61}$ This system was therefore investigated by simulating a water/methanol mixture (50:50) with the COMPASS force field. ${ }^{40}$ Figure 3 shows the $\phi_{M D}(\mathrm{r})$ functions for pure methanol, pure water, and for a mixed system of water-methanol, which reveals all correlations decay in a similar manner as separations approach $20 \AA$. The onset of the water-methanol correlations occurs about halfway between that for the pure systems, which is a consequence of the size of the molecules. This shows that permanent dipole moments adapt to correlate with other permanent dipole moments in different molecules. An interesting extension of these simulations will be an investigation of the entropy changes in $\phi_{M D}(\mathrm{r})$ for a transition from pure to mixed systems, which is currently under investigation.

Recently, the influence of various atomic ions on water's long-range dipolar correlations was reported, ${ }^{62}$ where it was found that the presence of a single ion can modulate the pair correlations that contribute to the hydrophobic effect. ${ }^{36}$ Furthermore, the ion's presence induces new correlations between permanent dipole moments and the ion itself; the free energy of which results in a Hofmeister series for different ions, ${ }^{62}$ demonstrating specific ion effects with respect to dipole-ion correlations. Here, the interest is on the structure and lengthscale of the perturbation of water's structure induced by an ion's presence, as found in similar dipole correlations to those in $\phi_{M D}(\mathrm{r})$.

To describe the correlations between a permanent dipole moment in water with an ion, only one dipole moment is needed, assuming the ion is not polarized, where the correlations are between water's dipole moment and the ion's center [see Eq. (8), for example]. The same treatment for $\phi_{M D}(\mathrm{r})$ applies, though many of the correlation functions $h_{l_{1} l_{2} l}$ now vanish. The water-ion correlations may be written in terms of

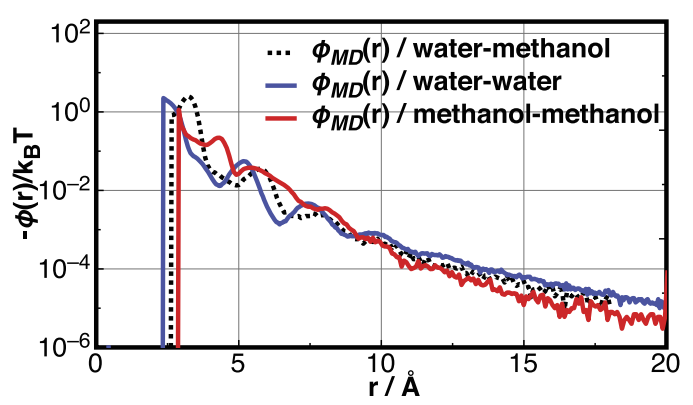

FIG. 3. The $\phi_{M D}(\mathrm{r})$ functions for a heterogeneous system of methanol and water (50:50) along with the $\phi_{M D}(\mathrm{r})$ functions for the respective pure systems. a potential of mean force $\psi_{M D}(\mathrm{r})$, given as

$$
\psi_{M D}(r)=-k_{B} T \frac{1}{2\left(g_{\alpha w}(r)\right)^{2}} \sum_{n=1}^{\infty}\left(h_{n 0 n}(r)\right)^{2},
$$

where $\alpha$ and $w$ denote an ion and water, respectively. The correlations between water's dipole moment and the chloride ion, as determined from Eq. (18), are evaluated from simulated trajectories of a single chloride ion in 2112 water molecules with the COMPASS force field ${ }^{63}$ and are shown in Fig. 4.

Similar to correlations between two dipole moments (Fig. 1), Fig. 4 shows correlations between water's dipole moment and a chloride ion that exist outwards of $20 \AA$ away from the chloride ion, where it is still non-vanishing, with obvious oscillations in structure up until $12 \AA$ of separation. The initial peaks and troughs in the $\psi_{M D}(\mathrm{r})$ function appear to approximately match those in the $g(\mathrm{r})$, but the oscillations in $\psi_{M D}(\mathrm{r})$ continue past the 2 nd solvation shell in the $g(\mathrm{r})$, where it is possible to see what appears to be a 4 th "shell" in the $\psi_{M D}(\mathrm{r})$ function at about $10 \AA$. It is important to note that the structural order that is found in the $\psi_{M D}$ (r) functions is a result of strong orientational correlations between solvating water and the ion. This cannot be found in the $g(\mathrm{r})$ function, which is a function of density, rather than orientation.

We performed a further simulation of a charge-neutral system ( $\mathrm{NaCl}$ in water), which produced the same correlations as that in Fig. 4, clarifying that the $\psi_{M D}(\mathrm{r})$ function is not affected by the lack of a counter ion (see supplementary material).

Following the treatment by Besford et al. ${ }^{62}$ the contribution of $\psi_{M D}(\mathrm{r})$ to the solvation free energy of the chloride ion may be given as

$$
G_{\psi_{M D}}=4 \pi \rho \int_{0}^{\infty} d r r^{2} \psi_{M D}(r) g_{\alpha w}(r)
$$

which for the system in Fig. 4 is evaluated to be $-26 \mathrm{~kJ} / \mathrm{mol}$, in reasonable agreement for the same correlations for a chloride ion in the SPC/E water model $(-31 \mathrm{~kJ} / \mathrm{mol})$. Whilst a large portion of the solvation free energy of an ion, which for chloride in water is about $-311 \mathrm{~kJ} / \mathrm{mol},{ }^{62}$ originates from the electrostatic forces related to the charge of the ion, ${ }^{64}$ the portion originating from $G_{\psi_{M D}}$ is still significant in magnitude. Crucially, the solvation free energy from $G_{\psi_{M D}}$ is specific to the ion's perturbation of water's structure that varies in a Hofmeister series for different ions, ${ }^{62}$ thus demonstrating a specific ion effect with respect to dipole-ion correlations in an ionic aqueous solution.

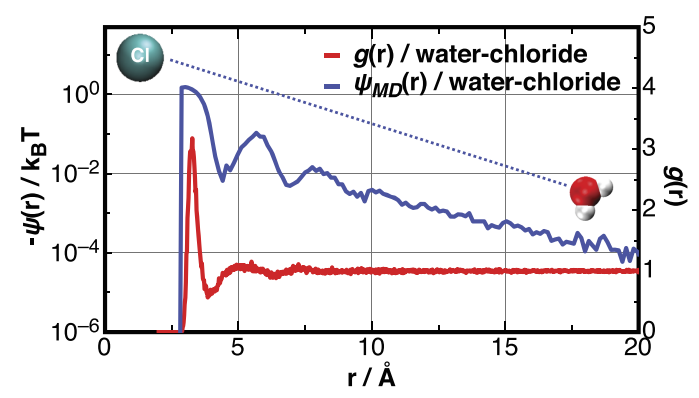

FIG. 4. MD results for the long-range correlations between water's dipole moment and a single chloride ion, $\psi_{M D}(\mathrm{r})$ (blue line), and the RDF between water's oxygen and the ion (red line). 
The long-range correlations between thermally rotating water molecules and the chloride ion are of a low energy, which are unlikely to be easily determined by experiment, particularly when searching for ion-specific effects. For example, femtosecond elastic second harmonic scattering (fs-ESHS) measurements have been used to probe structural changes in water induced by an ion's presence, which have suggested that specific ion effects are only found at high ionic concentrations $(0.1 \mathrm{M}-1 \mathrm{M}){ }^{22}$ The ion-induced changes in water structure are modulated differently depending on the ion, as observed through the $\psi_{M D}(\mathrm{r})$ correlations and the resulting free energy; however, the structural differences are subtle, ${ }^{62}$ hence it is unclear at this point how this may relate to fs-ESHS measurements. From a purely structural perspective, pressure perturbation calorimetry measurements have shown that an ion can affect water structure beyond one or two molecule hydration layers, ${ }^{65}$ consistent with the $\psi_{M D}(\mathrm{r})$ correlations. This demonstrates how subtle changes in water's structure induced by an ion's presence, with the associated free energy, may modulate the solvation capacity of water around solutes. The modulation of water's properties will likely have consequences for the solvation of complex solutes in aqueous systems, such as stabilizing solvated proteins ${ }^{66}$ where water plays an important role in protein solvation dynamics. ${ }^{35}$

Recently, fs-ESHS was used to detect changes in the orientational order of water molecules in bulk electrolyte solutions over nanoscopic length scales, ${ }^{67}$ where it was found that the fs-ESHS response in the concentration range of $1 \mu \mathrm{M}-100$ $\mathrm{mM}$ arises from water-water correlations induced by ions. This fs-ESHS observation was captured by a simple meanfield model that treats water molecules as non-interacting dipoles orientated by the electrostatic field of ions. ${ }^{68}$ The $\psi_{M D}(\mathrm{r})$ functions show that the ion's presence creates a new structural order with respect to water-ion correlations; however, it is not clear what consequence this has for water-water correlations.

Figure 5 shows the $\phi_{M D}(\mathrm{r})$ functions that were evaluated between water molecules in the simulation in Fig. 4, where at least one water molecule is within the $1 \mathrm{st}$, $2 \mathrm{nd}$, or $3 \mathrm{rd}$ solvation shells around the chloride ion. It is clear that water molecules within the 1st solvation shell experienced enhanced interactions with other waters, which is also experienced by waters in the 2 nd and 3 rd solvation shells, but to a lesser extent.

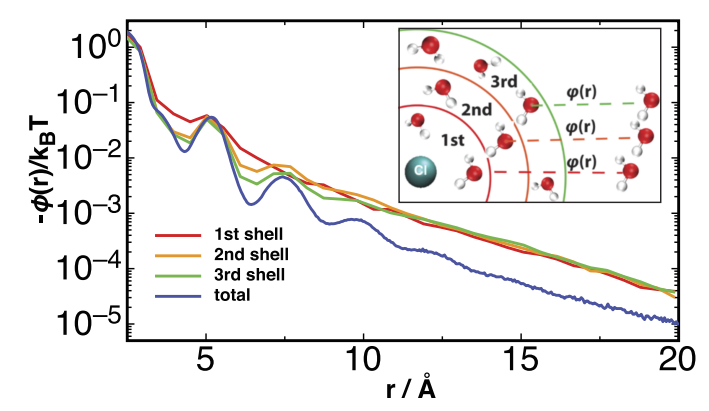

FIG. 5. The $\phi_{M D}$ (r) functions between water molecules within the simulated system containing a chloride ion, where at least one water is in the first (red), second (orange), and third (green) solvation shells of the chloride ion, and the total $\phi_{M D}(\mathrm{r})$ for all water molecules in the system. The solvation shells are

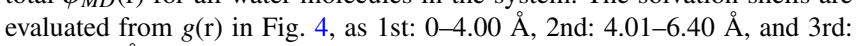
6.41-8.40 ̊.
However, when comparing to the total $\phi_{M D}(\mathrm{r})$ function across the entire system, the waters within the first three solvation shells have an enhanced long-ranged interaction with other waters, especially between about $8 \AA$ to almost $20 \AA$. These results demonstrate a significant modulation of the water-water interaction by the presence of a chloride ion. This is analogous to other work which has shown the modulation of waterwater interactions by the presence of fluoride, iodide, lithium, and cesium ions; ${ }^{62}$ however, here we have shown a longerranged modulation of the interaction caused by a chloride ion.

Finally, the application of Lifshitz theory to biological systems, as a part of the Derjaguin, Landau, Verwey, and Overbeek theory, ${ }^{69}$ has long been hindered by its inability to account for specific ion effects. ${ }^{70}$ This is attributed to the inconsistent separation of forces into electrostatic (non-linear PoissonBoltzmann description) and van der Waals forces (linear Lifshitz theory) ${ }^{71,72}$ By combining a rigorous analysis of pair correlations for the long-range dipolar order of polar liquids, with comparisons to dispersion forces and ion-induced changes in water's structure and water-water interactions, our results point toward a unified understanding of polar liquid structure with dipolar dispersion forces that uniquely encompass specific ion effects.

\section{v. CONCLUSIONS}

In conclusion, we have performed a rigorous analysis of liquid structure for various pure polar liquids, a mixed system of water and methanol, and an ionic aqueous system of chloride in water. Unique structural order was found for each system which extends at least up to $20 \AA$ of separation. This structure was compared to a dispersion force that arises from local co-operative enhancement of dipole moments, where strong agreement was found, showing that the unique longrange order is a result of a dipolar dispersion force between thermally rotating dipole moments. For comparison, Lifshitz theory of dipolar dispersion forces was compared with the structural order whereby the theory was validated for polar liquids that do not have local dipolar order, as quantified by the Kirkwood $g$-factor, $g_{K}$. For liquids that do have local dipole correlations (i.e., $g_{K}>1$ ), specifically liquid water, Lifshitz theory underestimates the force by about a factor of 5-10, therefore demonstrating that the dipolar dispersion force that leads to increased structure in liquid water is missed by Lifshitz theory of van der Waals forces.

A similar set of pair correlation functions was applied to a large system of water solvating a single chloride ion, where orientational correlations in structure were found at $20 \AA$ of separation, demonstrating a significant range of influence of an ion on water's structure that cannot be found by density distribution functions. Furthermore, we found that waters within the 1st, 2nd, and 3rd solvation shells of a chloride ion exhibit significantly enhanced dipolar interactions, particularly with waters at larger distances of separation.

By combining a thorough analysis of polar liquid structure with dispersion forces, our results provide new insight into the mechanisms that lead to long-range order in polar liquids. When accompanied with the observed ion-induced structural 
changes in water, our work points toward a unified theoretical framework of dispersion forces and specific ion effects in liquids, which may lead to a more robust understanding of complex solvation phenomena.

\section{SUPPLEMENTARY MATERIAL}

See supplementary material for simulations that investigate the effect of the electrostatic cutoff and larger system sizes on $\phi_{M D}(\mathrm{r})$ functions, as well as the simulated properties of each liquid, $\psi_{M D}$ correlations for a charge neutral simulation of $\mathrm{NaCl}$ in water, and the complete list of basis functions that contribute to $\phi_{M D}(\mathrm{r})$.

\section{ACKNOWLEDGMENTS}

We thank Dr. Angus Gray-Weale for initial discussions, Dr. Robert Falconer for advice on PPC measurements, and two anonymous referees for constructive criticism. A.J.C. and I.Y. acknowledge the generous allocation of high-performance computational resources from the Pawsey Supercomputing Centre and Melbourne Bioinformatics. Q.A.B. gratefully acknowledges The University of Melbourne for financial support.

${ }^{1}$ F. Hofmeister, Arch. Exp. Pathol. Pharmakol. 24, 247 (1888).

${ }^{2}$ B. W. Ninham and V. Yaminsky, Langmuir 13, 2097 (1997).

${ }^{3}$ V. Mazzini and V. S. J. Craig, Curr. Opin. Colloid Interface Sci. 23, 82 (2016).

${ }^{4}$ Q. A. Besford, M. Liu, J. K. Beattie, and A. Gray-Weale, J. Polym. Sci., Part B: Polym. Phys. 54, 948 (2016).

${ }^{5}$ G. W. Stevens, H. R. C. Pratt, and D. R. Tai, J. Colloid Interface Sci. 136, 470 (1990).

${ }^{6} \mathrm{~J}$. N. Israelachvili, Intermolecular and Surface Forces, 3rd ed. (Academic Press, Inc. Elsevier, London, 2011).

${ }^{7}$ S. Marcelja, J. D. Mitchell, and B. W. Ninham, J. Chem. Soc., Faraday Trans. 73, 630 (1977).

${ }^{8}$ J. Israelachvili and H. Wennerström, Nature 379, 219 (1996).

${ }^{9}$ F. London, Trans. Faraday Soc. 33, 8 (1937).

${ }^{10}$ B. M. Pettitt and P. J. Rossky, J. Chem. Phys. 77, 1451 (1982).

${ }^{11}$ P. J. Rossky, Annu. Rev. Phys. Chem. 36, 321 (1985).

${ }^{12}$ J. G. Kirkwood, Trans. Faraday Soc. 42, A007 (1946).

${ }^{13}$ G. Oster and J. G. Kirkwood, J. Chem. Phys. 11, 175 (1943).

${ }^{14}$ J. G. Kirkwood, J. Chem. Phys. 7, 911 (1939).

${ }^{15}$ J. Israelachvili and P. McGuiggan, Science 241, 795 (1988).

${ }^{16}$ J. N. Israelachvili and D. Tabor, Proc. R. Soc. A 331, 19 (1972).

${ }^{17}$ V. Parsegian and B. Ninham, Nature 224, 1197 (1969).

${ }^{18}$ R. Mancinelli, A. Botti, F. Bruni, M. A. Ricci, and A. K. Soper, Phys. Chem. Chem. Phys. 9, 2959 (2007).

${ }^{19}$ S. Myneni, Y. Luo, L. Naslund, M. Cavalleri, L. Ojamae, H. Ogasawara, A. Pelmenschikov, P. Wernet, P. Vaterlein, C. Haske, Z. Hussain, L. Pettersson, and A. Nilsson, J. Phys.: Condens. Matter 14, L213 (2002).

${ }^{20}$ H. J. Bakker and J. L. Skinner, Chem. Rev. 110, 1498 (2010).

${ }^{21}$ R. Bersohn, Y. Pao, and H. L. Frisch, J. Chem. Phys. 45, 3184 (1966).

${ }^{22}$ Y. Chen, H. I. I. Okur, C. Liang, and S. Roke, Phys. Chem. Chem. Phys. 19, 24678 (2017).

${ }^{23}$ K. B. Eisenthal, Chem. Rev. 96, 1343 (1996).

${ }^{24}$ A. Rahman and F. H. Stillinger, J. Chem. Phys. 55, 3336 (1971).

${ }^{25}$ F. H. Stillinger, J. Chem. Phys. 60, 1545 (1974).

${ }^{26}$ C. Y. Lee, J. A. M. J. Rossky, and J. A. Mccammon, J. Chem. Phys. 80, 4448 (1984).

${ }^{27}$ K. Voïtchovsky, D. Giofrè, J. José Segura, F. Stellacci, and M. Ceriotti, Nat. Commun. 7, 13064 (2016).

${ }^{28}$ J. Grdadolnik, F. Merzel, and F. Avbelj, Proc. Natl. Acad. Sci. U. S. A. 114, 322 (2017).

${ }^{29}$ Y. Liu and J. Wu, J. Chem. Phys. 139, 041103 (2013).

${ }^{30} \mathrm{G}$. Mathias and P. Tavan, J. Chem. Phys. 120, 4393 (2004).
${ }^{31}$ U. Baul, J. M. P. Kanth, R. Anishetty, and S. Vemparala, J. Chem. Phys. 144, 104502 (2016).

${ }^{32}$ C. Zhang and G. Galli, J. Chem. Phys. 141, 084504 (2014).

${ }^{33}$ S. H. Lee and P. J. Rossky, J. Chem. Phys. 100, 3334 (1994).

${ }^{34}$ J. M. P. Kanth, S. Vemparala, and R. Anishetty, Phys. Rev. E 81, 021201 (2010).

${ }^{35}$ M. C. Bellissent-Funel, A. Hassanali, M. Havenith, R. Henchman, P. Pohl, F. Sterpone, D. Van Der Spoel, Y. Xu, and A. E. Garcia, Chem. Rev. 116, 7673 (2016).

${ }^{36}$ M. Liu, Q. A. Besford, T. Mulvaney, and A. Gray-Weale, J. Chem. Phys. 142, 114117 (2015).

${ }^{37}$ J. P. Hansen and I. R. McDonald, Theory of Simple Liquids, 2nd ed. (Academic Press, London, 1986).

${ }^{38}$ C. Gray and K. Gubbins, Theory of Molecular Fluids (Oxford University Press, 1984).

${ }^{39}$ J. P. Hansen and I. R. McDonald, Theory of Simple Liquids, 2nd ed. (Academic Press, London, 2002).

${ }^{40}$ H. Sun, J. Phys. Chem. B 102, 7338 (1998).

${ }^{41}$ J. Guo, B. L. Tardy, A. J. Christofferson, Y. Dai, J. J. Richardson, W. Zhu, M. Hu, Y. Ju, J. Cui, R. R. Dagastine, I. Yarovsky, and F. Caruso, Nat. Nanotechnol. 11, 1105 (2016).

${ }^{42}$ G. Yiapanis, A. J. Christofferson, M. Plazzer, M. P. Weir, E. L. Prime, G. G. Qiao, D. H. Solomon, and I. Yarovsky, Langmuir 29, 14451 (2013).

${ }^{43}$ A. Ignaczak, J. A. N. F. Gomes, and M. N. D. S. Cordeiro, Electrochim. Acta 45, 659 (1999).

${ }^{44}$ D. Lide, Handbook of Chemistry and Physics, 87 th ed. (Taylor \& Francis, Boca Raton, FL, 2007).

${ }^{45}$ S. Plimpton, J. Comput. Phys. 117, 1 (1995).

${ }^{46}$ BIOVIA Materials Studio Version 8.0, 2014.

${ }^{47}$ A. Dequidt, J. Devemy, and A. A. H. Pádua, J. Chem. Inf. Model. 56, 260 (2015).

${ }^{48}$ G. Lamoureux, E. Harder, I. Vorobyov, B. Roux, and A. MacKerell, Jr., Chem. Phys. Lett. 418, 245 (2006).

${ }^{49}$ S. Y. Noskov, G. Lamoureux, and B. Roux, J. Phys. Chem. B 109, 6705 (2005).

${ }^{50}$ D. P. Shelton, J. Chem. Phys. 143, 134503 (2015).

${ }^{51}$ K. Laasonen, M. Sprik, M. Parrinello, and R. Car, J. Chem. Phys. 99, 9080 (1993).

${ }^{52}$ E. Lifshitz, Sov. Phys. JETP 2, 73 (1956).

${ }^{53}$ I. Dzyaloshinskii, E. Lifshitz, and L. Pitaevskii, Sov. Phys.-Usp. 73, 381 (1961).

${ }^{54}$ D. C. Prieve and W. B. Russel, J. Colloid Interface Sci. 125, 1 (1988).

${ }^{55}$ E. Sabisky and C. Anderson, Phys. Rev. A 7, 790 (1973).

${ }^{56}$ J. Israelachvili and R. Pashley, Nature 300, 341 (1982).

${ }^{57}$ E. E. Meyer, K. J. Rosenberg, and J. Israelachvili, Proc. Natl. Acad. Sci. U. S. A. 103, 15739 (2006).

${ }^{58}$ H. K. Christenson and P. M. Claesson, Adv. Colloid Interface Sci. 91, 391 (2001).

${ }^{59}$ Y. Marcus, J. Solution Chem. 21, 1217 (1992).

${ }^{60}$ M. S. Skaf and B. M. Ladanyi, J. Chem. Phys. 102, 6542 (1995).

${ }^{61}$ H. Yu, D. P. Geerke, H. Liu, and W. F. Van Gunsteren, J. Comput. Chem. 27, 1494 (2006).

${ }^{62}$ Q. A. Besford, M. Liu, and A. Gray-Weale, Phys. Chem. Chem. Phys. 18, 14949 (2016).

${ }^{63}$ J. Guo, J. J. Richardson, Q. A. Besford, A. J. Christofferson, Y. Dai, C. W. Ong, B. L. Tardy, K. Liang, G. H. Choi, J. Cui, P. J. Yoo, I. Yarovsky, and F. Caruso, Langmuir 33, 10616 (2017).

${ }^{64}$ M. A. Vorotyntsev and A. A. Rubashkin, Phys. Chem. Liq. 55, 141 (2017).

${ }^{65}$ J. W. Bye and R. J. Falconer, Phys. Chem. Chem. Phys. 17, 14130 (2015).

${ }^{66}$ J. W. Bye and R. J. Falconer, Protein Sci. 22, 1563 (2013).

${ }^{67}$ Y. Chen, H. I. Okur, N. Gomopoulos, C. Macias-Romero, P. S. Cremer, P. B. Petersen, G. Tocci, D. M. Wilkins, C. Liang, M. Ceriotti, and S. Roke, Sci. Adv. 2, e1501891 (2016).

${ }^{68}$ D. M. Wilkins, D. E. Manolopoulos, S. Roke, and M. Ceriotti, J. Chem. Phys. 146, 181103 (2017).

${ }^{69}$ E. Verwey and J. T. Overbeek, Theory of the Stability of Lyophobic Colloids: The Interaction of Sol Particles Having an Electric Double Layer (Elsevier, New York, 1948).

${ }^{70}$ B. W. Ninham, R. M. Pashley, and P. L. Nostro, Curr. Opin. Colloid Interface Sci. 27, 25 (2017).

${ }^{71}$ M. Boström, D. R. M. Williams, and B. W. Ninham, Phys. Rev. Lett. 87, 168103 (2001).

${ }^{72}$ B. W. Ninham, T. T. Duignan, and D. F. Parsons, Curr. Opin. Colloid Interface Sci. 16, 612 (2011). 